\title{
Evaluación del aprendizaje, institucional y de amplia escala en el contexto escolar
}

\author{
Institutional and large-scale learning assessment in the school context \\ Avaliação da aprendizagem institucional e de grande escala no contexto escolar
}

Recibido: 27/12/2021 | Revisado: 02/01/2022 | Acepto: 06/01/2022| Publicado: 10/01/2022

\author{
Maria Aparecida Vieira de Melo \\ ORCID: https://orcid.org/0000-0001-6288-9405 \\ Universidade Federal do Rio Grande do Norte, Brasil \\ E-mail: m_aparecida_v_melo@hotmail.com \\ Ricardo Santos de Almeida \\ ORCID: https://orcid.org/0000-0003-1266-2557 \\ Universidade Federal de Santa Maria, Brasil \\ Universidad Interamericana, Paraguay \\ E-mail: ricardosantosal@gmail.com
}

\begin{abstract}
Resumen
El enfoque sistemático sobre la evaluación del aprendizaje, institucional de amplia escala planea analizar lo que viene sendo señalado en esta perspectiva triadic en el contexto escolar, pues los instrumentos metodológicos deben de este modo atravesar tal finalidad evaluativa. De esta manera, iremos operar con el enunciado de la evaluación en relación a la evaluación del aprendizaje, institucional y amplia escala al fin y al cabo desea medir el aprendizaje en una dimensión micro y macro. ¿La cuestión principal, es cuál la finalidad de la evaluación en el contexto escolari Tenemos por finalidad identificar los despliegues de la evaluación en el contexto escolar como herramienta de promoción del desarrollo y fortalecimiento de la institución que se dedica en educar para un cierto fin, comprender las funciones de la evaluación en el contexto escolar y problematizar la finalidad de la evaluación en amplia escala así como la institucional. Como hallazgos, podemos señalar que evaluación del aprendizaje visa el aprendizaje de los alumnos, la evaluación institucional, el desarrollo de la institución y la evaluación en amplia escala corrobora con el Índice de Desenvolvimento da Educação Básica (IDEB), lo cual mide la calidad de la educación.
\end{abstract}

Palabras clave: Sistema de evaluación; Educación; Políticas educacionales.

\begin{abstract}
The systematic approach to the evaluation of learning, institutional on a wide scale, plans to analyze what has been indicated in this triadic perspective in the school context, since the methodological instruments must in this way cross such an evaluative purpose. In this way, we will operate with the statement of evaluation in relation to the evaluation of learning, institutional and broad scale after all, you want to measure learning in a micro and macro dimension. The main question is what is the purpose of the evaluation in the school context? We aim to identify the deployment of the evaluation in the school context as a tool to promote the development and strengthening of the institution that is dedicated to educating for a certain purpose, understand the functions of evaluation in the school context and problematize the purpose of evaluation on a wide scale as well as the institutional one. As findings, we can point out that learning evaluation aims at student learning, institutional evaluation, institution development and wide-scale evaluation corroborates with the Índice de Desenvolvimento da Educação Básica (IDEB), which measures quality of Education.
\end{abstract}

Keywords: Evaluation system; Education; Educational policies.

\section{Resumo}

A abordagem sistemática da avaliação da aprendizagem, institucional em larga escala, pretende analisar o que tem sido indicado nesta perspectiva triádica no contexto escolar, uma vez que os instrumentos metodológicos devem, assim, cruzar tal finalidade avaliativa. Dessa forma, vamos operar com o enunciado da avaliação em relação à avaliação da aprendizagem, em escala institucional e ampla, afinal, pretende-se medir a aprendizagem numa dimensão micro e macro. A questão central é: qual a finalidade da avaliação no contexto escolar? Pretendemos identificar a implantação da avaliação no contexto escolar como uma ferramenta para promover o desenvolvimento e o fortalecimento da instituição que se dedica a educar para um determinado fim, compreender as funções da avaliação no contexto escolar e problematizar o propósito da avaliação tanto em larga escala como institucional. Como constatações, podemos apontar que a avaliação da aprendizagem visa a aprendizagem do aluno, a avaliação institucional, o desenvolvimento da instituição e a avaliação em larga escala corrobora com o Índice de Desenvolvimento da Educação Básica (IDEB), que mede a qualidade da Educação.

Palavras-chave: Sistema de avaliação; Educação; Políticas educacionais. 


\section{Introducción}

Evaluar es un problema. Sí, la evaluación en el contexto escolar ni siempre es fácil de se hacer. De ahí que varios instrumentos son criados para subvencionar en el proceso de adquisición del conocimiento que debe ser medido delante el acto de la evaluación. De esta manera, resaltamos que hay una región discursiva en que la evaluación está direccionada que perspectiva al fin y al cabo el desarrollo, sea él, en relación a el aprendizaje del educando, al desempeño de la institución y el desempeño de los estudiantes y de la institución simultáneamente debido a la evaluación en amplia escala. Así comprendemos que la evaluación asume un papel fundante en acto de venir a ser del desarrollo en el contexto escolar.

Muchas son las formas de evaluar, las cuales transcenden el instrumento examen. Sin embargo, para que la evaluación deje de ser un "bicho de siete cabezas" y que amenaza a todos los estudiantes, es necesario que se tenga como aliada del desarrollo en el contexto escolar. Pues como sabemos, cada tipo de evaluación corresponde a una finalidad específica, la cual iremos comprender más adelante. Así, no podemos tener la evaluación como instrumento castigador, amenazador y amedrentador que sea así considerado por los estudiantes, es necesario ampliar la visión acerca de la evaluación relativa a la finalidad que ella establece en el contexto escolar.

De este modo, iremos operar con las categorías y conceptos evaluación del aprendizaje, evaluación institucional y evaluación en amplia escala para analizarmos la función que cada una desempeña en el contexto escolar y, más específicamente, iremos identificar los desplazamientos que la evaluación promove en el desempeño de los estudiantes, reconocer cuales son las políticas educacionales que la evaluación institucional y en amplia escala están acostadas y por fin explicitar la implicación que la evaluación tiene en la calidad de la educación. Partiremos de la curiosidad en saber: ¿Cómo la evaluación tiene ejercido su papel social en el contexto escolar $i$ Pues, de este modo, iremos explicitar los hallazgos que en conjunto de las cosas dichas y escritas están puestas a partir de los despliegues de la evaluación.

Para dicha metodología y un análisis del discurso arqueológico (AAD) de Michel Foucault (2008), que trata de mapear las fuentes, desenterrar fragmentos de texto y explicar los atributos en torno a las categorías de evaluación del aprendizaje, evaluación institucional a gran escala. Además, una metodología es de carácter cualitativo que tiene como objetivo analizar los discursos de los puestos de evaluación y sus derivados.

No obstante, señalamos que la finalidad de la evaluación planea el desempeño en un doble movimiento, del aprendizaje de los estudiantes y la calidad en proceso de enseñanza de las instituciones que son evaluadas a partir de la evaluación en amplia escala que promove el índice de desarrollo de la educación tanto básica cuanto superior en que es concerniente al princípio de calidad de la educación.

\section{Evaluación del Aprendizaje: los Nexos Pedagógicos}

El abordaje sobre la evaluación del aprendizaje interesa en los procesos formativos que planean el desempeño de los estudiantes. Así, iremos profundizar en las camadas del lenguaje para analizarmos los despliegues de la evaluación del aprendizaje a respeto de los tipos de evaluación, el papel de cada tipo y la finalidad de la evaluación del aprendizaje. Sabemos que hay varios instrumentos de evaluación que atravesa la sistematización del conocimiento.

A lo largo del tiempo, la evaluación va sendo cambiada a depender de la manera como es concebida tanto por el sistema de enseñanza cuanto por la forma como es considerada por el educador en sala de clase. De este modo, sabemos que la evaluación es caracterizada como evaluación diagnóstica, sumativa y formativa, segundo (Luckesi, 2000). Así, sobre la evaluación sumativa está puesto que "La evaluación sumativa es una evaluación muy general, que sirve como punto de apoyo para atribuir notas, clasificar el alumno y transmitir los resultados en términos cantitativos, hecha en el final de un período" (Bloom et al., 1983, p.100). O sea, este tipo de evaluación planea el resultado, y no el proceso de adquisión del conocimiento. Cuando tratamos la evaluación como clasificatoria estamos dando énfasis sólamente a la clasificación del estudiante sin 
considerar el todo de la parte componen la parte toda, pues como (Hoffmann, 1998, p. 57) alertarnos que la evaluación no debe ser clasificatoria, pues ella "considera las tareas en una linealidad, sin la articulación de una con la otra, lo que las convierte independientes y estáticas. Esto es, una práctica evaluativa tradicional, estanque que se engancha al instrumento evaluativo y al rato de la evaluación, desconsiderando todas las demás etapas que atravesan el aprendizaje. En esta perspectiva, (Luckesi, 2005, p. 01) deprende por evaluación sumativa como resultado, pues "eses resultados siempre serán positivos se efectivamente fueron construidos como resultados deseados". Así, los resultados se planeados para seren alcanzados rompen con la dimensión de la evaluación clasificatoria, cuya es una finalidad de la evaluación sumativa de carácter tradicional.

Al aprofundizarmos en las dimensiones de la evaluación diagnóstica, identificamos como base para sabermos el nivel de la clase y así planear con la finalidad de ampliar los saberes de los sujetos. Esto es, se hace un diagnóstico general de la realidad en que se encuentran los estudiantes. En esta lógica, señalamos lo que está puesto por (Machado 1995, p. 33) al considerar que "La evaluación diagnóstica posibilita al educador y educando detectaren, a lo largo del proceso de aprendizaje, sus fallas, desvíos, sus dificultades, a tiempo de redireccionar los medios, los recursos, las estrategias y procedimientos en la orientación deseada". De esta manera, la evaluación diagnóstica asume función doble, cual sea la de posibilitar al educador aprehender la realidad y hacer una planificación que parta de la realidad encontrada para profundizarla, viabilizando acciones concretas para promover procesos educativos concernientes para el aprendizaje de los alumnos (Perrenoud, 2008; Hadji, 2001).

Ya evaluación formativa/mediadora corresponde al proceso continuo formativo de los estudiantes, pues no se engancha al empezo, ni al medio y ni al fin del proceso evaluativo, pero sí el su continuum. De haí es necesario que "cambie la acción evaluativa en un rato de acción del aprendizaje y todavía en un rato de acción-reflexión-acción que apoyará futuras intervenciones concernientes al currículo y sus despliegues en el proyecto político pedagógico de un curso" (Carminatti \& Borges, 2020, p.174).

Compréndese que la evaluación formativa/mediadora es diferente de la evaluación sumativa, la cual se ocupa de la clasificación, punición, control y amenaza de se ganar o perder, debido a la dimensión punitiva que asume. Sin embargo, la evaluación formativa/mediadora es trascendental al que concierne a los procesos de enseñanza - aprendizaje, pues la evaluación es un artefacto de la promoción del conocimiento por medio de la reflexión-acción-reflexión. Entiéndese por evaluación mediadora como un "proceso de permanente cambio de mensajes y de significados, un proceso interactivo, dialógico, espacio de encuentro y de confronto de ideas entre educador y educando en busca de patamares calitativamente superiores de saber" (Hoffmann, 2009, p.76). Pues hay una complejidad en el acto de evaluar que no debe el proceso ser reducido tan sólamente a dar nota. Tenendo en vista que la dinámica de la evaluación es compleja, pues necesita ajustarse a los "trayectos individuales de aprendizaje que se dan en el colectivo y, por lo tanto, en múltiples y distintas direcciones" (Hoffmann, 2009, p.78).

Así, podemos señalar como la evaluación se despliega en sus modalidades y funciones que ejerce de acuerdo con su finalidad y rato evaluativo como lo sugieren Carvalho e Ribeiro (2020, p.4) con el objetivo de fortalecer el perfil docente profesional en Brasil., tal como podemos visualizar abajo: 
Cartel 1: Visualizando los tipos de evaluación.

\begin{tabular}{|c|c|c|c|}
\hline Modalidad (tipo) & Función & Propósito (Para qué usar) & Época (Cuándo aplicar) \\
\hline Diagnóstica & Diagnosticar & $\begin{array}{l}\text { Averiguar la presencia o ausencia de requisitos } \\
\text { previos para nuevos aprendizajes. Detectar } \\
\text { dificultades específicas de aprendizaje, } \\
\text { intentando identificar sus causas. }\end{array}$ & $\begin{array}{l}\text { Inicio de año o semestre lectivos, } \\
\text { o en inicio de una unidad de } \\
\text { enseñaza. }\end{array}$ \\
\hline Sumativa & Clasificar & $\begin{array}{l}\text { Clasificar los resultados de aprendizaje } \\
\text { alcanzados por los alumnos, de acuerdo con } \\
\text { niveles de aprovechamiento establecidos. }\end{array}$ & $\begin{array}{l}\text { Al final de un año o semestre } \\
\text { lectivos, o al final de una unidad } \\
\text { de enseñaza. }\end{array}$ \\
\hline Formativa & Controlar & $\begin{array}{l}\text { Constatar se los objetivos establecidos fueron } \\
\text { alcanzados por los alumnos. Proveer datos para } \\
\text { perfeccionar el proceso enseñaza y aprendizaje }\end{array}$ & $\begin{array}{l}\text { Durante el año lectivo, esto es, a } \\
\text { la lo largo del proceso enseñaza y } \\
\text { aprendizaje. }\end{array}$ \\
\hline
\end{tabular}

Fuente: Ferreira (2003).

Con este cartel síntesis, Ferreira explicita de modo general el tipo y la función que cada evaluación ejerce en el rato en que sucede la evaluación. De esta manera, la planificación de enseñanza es crucial para que por medio de la investigación de los procesos educativos y de los instrumentos metodológicos sean definidos el mejor tipo de evaluación que ofrece el aprendizaje, tal como reflexionamos, identificamos que la evaluación diagnóstica y la formativa/mediadora son las que más mejor se adecuan a las demandas del aprendizaje de los estudiantes, primando así, por reflexión-acción-reflexión en un movimiento doble tanto de la costumbre del profesor cuanto del aprendizaje de los alumnos, eludindo, por tanto, con la práctica de la evaluación punitiva, controladora y amenazadora, vertendo así para una evaluación democrática y emancipatoria de los procesos de adquisición del conocimiento en el contexto escolar.

\section{Evaluación Institucional: Delimitando la Territorialidad Formativa}

La evaluación institucional fecha de 1982 la cual surge mediante la necesidad de promover la superación del fracaso escolar del educando, tenendo en vista que él no es el único responsable por el fracaso escolar. Tenendo en vista que la propria institución de enseñaza también tiene responsabilidad conforme las prácticas de enseñaza que son subvencionadas, la gestión escolar que es establecida y la propuesta curricular que es mantenida.

Subrayamos, por tanto, que la evaluación institucional corresponde a las evaluaciones que son establecidas para que la institución pueda ser y tener un desempeño de mejor calidad. Sendo así, conforme la práctica discursiva sobre la evaluación institucional, está puesto por (Libâneo, 2012, p.01) que:

En 1982, la Andes - Asociación Nacional de los Docentes de la Enseñaza Superior - propuso la evaluación institucional como un recurso subsidiario de la mejora del desempeño de cada institución. En 1983, el MEC instituyó el PARU - Programa de Evaluación de la Reforma Universitaria. En 1993, fue criado el PAIUB - Programa de Evaluación Institucional de la Universidad Brasileña. En 1996, fue implantado el Examen Nacional de Curso, popularmente conocido por "Provão", que, en 2004, se cambió en SINAES - Sistema Nacional de Evaluación de la Enseñaza Superior. A respeto de fechas iniciales, seguimos por la Enseñaza Básica. El Sistema de Evaluación de la Enseñaza Básica - SAEB - fue criado en 1998, con su primera aplicación en 1990, que, por medio de la perspectiva de la evaluación de la educación en el país, sufrió los perfeccionamientos con el Examen Brasil (2005) y con el IDEB Índice de Desarrollo de la Educación Básica (2007). Por fin, llegamos a la Enseñaza Media, cuya expresión actual decurre de la denominación "Nuevo ENEM" (2009).

Así, averiguamos que historicamente la evaluación va se expandindo, de tal manera que promueva una reflexión sobre toda la institución de enseñaza. De este modo, las evaluciones son criadas de acuerdo con la finalidad en la dimensión micro (aprendizaje de los estudiantes) a la dimensión macro (sistema de educación, institución de enseñaza). Las pruebas y exámenes que son criados espaldan la evaluación institucional, pues los resultados confirman para desarrollar acciones de 
mejoría en la institución escolar, lo que es puesto en el programa de desarrollo institucional (PDI), en el proyecto político pedagógico del curso (PPPC).

Sendo así, comprendemos la evaluación institucional sendo de la responsabilidad de los sectores organizacionales y gestión de los sistemas de enseñaza. Objetiva colectar datos cantitativos y calitativos del colectivo escolar: alumnos, profesores, la estructura organizacional los recursos físicos y materiales, las prácticas de gestión, la productividad de la enseñaza, visando expresar y parecer valorativo sobre la institución y su desarrollo. Ya la evaluación académica, o científica, objetiva datos cantitativos del aprendizaje a través del acompañamiento de las políticas de la red escolar y de las escuelas, preocupándose en establecer índices calitativos del proceso educativo (Cuaderno Pedagógico, 2020, p.142).

Los datos que son colectados por los sectores organizacionales corroboran para que puedan ser tomadas decisiones sobre las acciones necesarias que atravesen la calidad de la educación ofrecida en la institución de enseñaza. Importa relatar que los datos cantitativos son mensurados calitativamente. Así, la calidad de la educación es puesta en evidencia, tenendo en vista los índices calitativos del proceso educativo.

Comprender la especificidad de la evaluación institucional es esencial, pues corresponde la realidad que se tiene y lo que se intenta cambiar, a partir del diagnóstico realizado, o sea, por medio de los datos colectados lo que hacer con el resultado. En esta perspectiva, señala (Gadotti, 2000, p.195) que:

La evaluación institucional no más es vista como un instrumento de control burocrático y centralizador, en conflicto con la autonomía. Ella está sendo institucionalizada como un proceso necesario de la administración de la enseñaza, como condición para la mejoría de la enseñaza y de la investigación y como exigencia de la democratización. Mismo así, ella encuentra resistencias y no se constituye en una práctica constante. Por eso, debe ser más instituida hasta tornarse en una solicitación explícita de las escuelas.

Sabendo que la evaluación institucional es tenida como un instrumento de control burocrático y centralizador, indagamos como podemos hacer para que se torne un instrumento que averigue los cambios que son necesarios en la institución y así se tome las decisiones pertinentes para promover una educación de calidad, desde la infraestructura de la institución al trabajo pedagógico de sus profesores. O sea, cambios de la acción micro a la macro.

Interesa mencionar que la complejidad de la evaluación institucional redefine el modus operandi de la institución de la enseñaza, lo que favorece para planificación de acuerdo con las exigencias de la institución, pues "[...] la evaluación institucional consiste en un emprendimiento sistemático que busca comprensión global de la Universidad, por el reconocimiento y por la integración de sus diversas dimensiones [...]”(Balzan; Sobrinho, 1995, p. 09), viabilizando así “[...] repensar el significado de la participación de los distintos actores en la vida y en el destino de las escuelas", haciendo jus, por lo tanto, la "dimensión colectiva del proyecto político pedagógico", para "reflexionar sobre sus potencialidades, vulnerabilidades y repercusiones en nivel de sala de clase, junto a los estudiantes" (Freitas et al, 2009, p.35).Conciébese, por lo tanto, la evaluación institucional como instrumento democrático, por requerir la participación de todos los sujetos colectivos en el contexto escolar.

Resaltase, por tanto, que la evaluación institucional tiene una finalidad cuya es promover mejoría y calidad en la educación institucional, por eso, que los datos colectados deben impulsar para los cambios que son necesarios y no simplemente almacenar dejándolos como fueron encontrados, tenendo en vista que "una evaluación institucional está volvida para producir transformaciones, esto es, evolución, crecimiento, desarrollo de la Universidad y no para cristalizala en el rato de su evaluación" (Masetto, 1990, p.10). Así debe ser, no importa se es en la universidad o se es en la escuela, el facto que los actos colectados apuntan los cambios que deben necesariamente seren hechos en el contexto escolar. 
A partir del rato que se da énfasis al objetivo de la evaluación institucional, los procesos educativos tanto en la esfera macro cuanto en la micro tenden a cambiar, así importa mencionar lo que está puesto sobre el objetivo de la evaluación institucional, a saber:

Debe se llevar al esfuerzo de los agentes de la acción educativa en la institución, en un proceso de autocrítica y transformación, comprometéndolos con el esbozo y con la ejecución de un proyecto de universidad donde la participación asegure el envolvimiento de aquellos que viven el cotidiano de la universidad, en su construcción y/o reconstrucción (Saul, 1990, p.18).

El empeño de los agentes de la acción educativa proyecta la gestión educativa en una perspectiva democrática, cual para allá de apuntar las necesidades emergentes de la institución señalado el estímulo de políticas públicas educacionales, tales como asistencia estudantil.

La universidad, a partir de la evaluación institucional, puede incentivar las transformaciones necesarias que conciernen los aspectos macros de su organicidad, así como de los procesos pedagógicos que atravesan la adquisición del conocimiento, o sea, las competencias y habilidades inherentes a los procesos formativos que penetran la formación de los estudiantes y su participación en el contexto escolar. Así sendo,

Sólamente considerando el papel de la universidad en la perspectiva de producir y transmitir conocimiento como medio para vivir mejor en un colectivo compartillado, podrán ser elegidos medios e instrumentos para una evaluación institucional que podrá ayudar en reales avanzos socioeducacionales, se fuera utilizada de manera que lleve a la construcción de visiones más integradas sobre su trayecto históricoinstitucional, en sus implicaciones concretas (Gatti, 2006, p.10).

La evaluación institucional visa a la construcción de perspectivas más integradas sobre la propria institución, la cual atravesa avanzos socioeducacionales. De tal manera, la elección de instrumentos para evaluación institucional es crucial para que trasnformaciones sucedan en la propria institución, pues la evaluación institucional de cuño participativo y formativo que posibilite un repensar de acciones, con vistas a la efectividad de la gestión y de la mejoría de la enseñaza (Décia e Argollo, 2010).

Porque la evaluación institucional asume un doble movimiento, pues ella informa y forma. Dicho en otras palabras, la evaluación institucional da subvenciones para que los procesos formativos sean establecidos para los profesores de la institución, así como la reconstrucción de cambios que la evaluación apunta seren necesarios. Tal como podemos constatar, en este fragmento, que:

Colocando el foco de la EI en el sentido formativo, más que un sencillo proceso informativo y estableciendo un carácter educativo, es que será posible instaurar un significado evaluativo con el objetivo de buscar evidenciar lo que es necesario cambiar y lo que es indispensable fortalecer y ampliar para la adquisición del suceso relacionado a la calidad de la educación superior (Ribeiro, 2013, p. 176).

Así la calidad de la educación puede ser medida a través de la evaluación institucional, pues tanto forma cuanto informa señalando los escopos que deben ser alcanzados en la institución. De esta manera, el trozo que explicita esta afirmación sobre la EI cumplir un doble movimiento, he aquí:

El aspecto formativo de la evaluación presupone que sus resultados sirvan de referencial para el perfeccionamiento de la gestión de las instituciones y que sean disponibilizadas las informaciones suficientes a la replanificación de las acciones e inversiones, con base en la evaluación interna y externa realizada periódicamente.[...] las informaciones resultantes del proceso de evaluación subvencionan la tomada de decisiones de los gestores sobre cambios 
necesarios en el curso, como la revisión de los currículos, de proyectos y de programas que vengan incurrir en nuevas prácticas y en tecnologías educacionales aplicadas a la necesaria formación del estudiante (Griboski, 2012, p.192, subrayos nuestros).

Además, la EI posibilita la formación de la identidad de la institución, así como perspectiva tomada de decisiones concernientes para que se pueda comparar con otras instituciones y superar las dificultades que la evaluación señala. Es un instrumento potencial para fomentar mejorías en la institución y calidad en la educación. Democráticamente, es un instrumento que incentiva la institución hacer una autoevaluación de si, en busca de se adecuar de elementos que posibiliten venir a ser más y mejor, con educación de calidad, cuando su infraestructura ofrecer el apoyo necesario para los procesos pedagógicos en el contexto escolar.

Es válido resaltar que EI no es direccionada tan sólamente para universidades, pero también para escuelas públicas que priman por una gestión democrática y que se ocupa con una educación de calidad. Así, se considera que para la EI suceder se debe tener en el contexto escolar gestores que así les gusten de participar de la comunidad escolar para allá de la construcción del proyecto político pedagógico, primando, por lo tanto, para la escuela como un todo a partir de la EI que también se hace con la comunidad escolar, sendo esta un instrumento importante que señala en que la escuela necesita cambiar, a través de los resultados, los gestores y profesores tomaren decisiones y ejecutaren planificaciones concernientes para promover la majoría en la escuela y la calidad en la educación. Así, “[...] las escuelas pueden y deben desarrollar mecanismos proprios de evaluación, que ayuden sus procesos de organización y gestión, con vistas al desarrollo de más calidad en la educación pública" (Souza \& Dittrich, 2012, p.45).

Señalamos que, en 2004, fue criado el Sistema Nacional de Evalución de la Educación Superior, a partir de la ley n ${ }^{\circ}$ 10.861, la cual se ocupa de la evaluación institucional, de los cursos y del desepeño de los estudiantes. Es un sistema de evaluación que hace uso de varios instrumentos evaluativos para que pueda dar cuenta de las especificidades de la institución como un todo, a saber los:

Instrumentos complementarios: autoevaluación, evaluación externa, ENADE, evaluación de los cursos de evaluación e instrumentos de información (censo y registro). Los resultados de las evaluaciones posibilitan dibujar un panorama de la calidad de los cursos e instituciones de educación superior en el país. Los procesos evaluativos son coordinados y supervisionados por la Comisión Nacional de Evaluación de la Educación Superior (Conaes). La operacionalización es de responsabilidad del INEP (Brasil, 2004).

Con tales instrumentos la EI corrobora para tomadas de decisiones de los gestores de la institución de la Enseñaza Superior, puesto que el SINAES fue criado para evaluar las Instituciones de la Enseñaza Superior. Así sendo, se percibe que hay un movimiento global de la evaluación, la cual posibilita la permanencia de la IES funcionar por medio del tamiz de la evaluación externa, a rigor el Ministerio de la Educación se ocupa en fomentar el credenciamiento o descredenciamiento a partir del resultado de la evalución externa.

Porque, comprendemos que EI es importante para un proceso de desarrollo en una perspectiva global de la institución, por viabilizar conocimiento sobre todos los aspectos de la institución, los cuales confirman para planificación de acciones que vengan a promover soluciones de los problemas diagnosticados, tenendo en vista que se convertir, al mismo tiempo, un instrumento democrático, donde la comunidad escolar/ académica puede medir los cambios necesarios y así a partir de la gestión educacional se tomar las medidas razonables para alcanzar la mejoría en la institución y la calidad en la educación, eso porque son indisociables calidad y mejoría.

Comprendendo la EI como acción global, notamos que los resultados, después de seren analizados y registrados en los informes promove el panorama de la situación de la institución, bien como señala en que los cambios se hacen necesarios. Así sendo, EI conforme la compresión de (Fernandes, 2001, p.15) es “[...] un proceso global, continuo y sistemático, 
competente y legítimo, participativo, que puede envolver agentes internos y externos en la formulación de las subvenciones para la mejoría de la calidad de la institución escolar". Significa, por lo tanto, que es un instrumento que atravesa las articulaciones entre los agentes internos y externos a la institución, así como hay una retroalimentación de la gestión escolar y de la gestión educacional en el contexto escolar/ académico. Por lo tanto, es de esta manera que la EI tanto forma cuanto informa.

Registra la importancia de la EI la participación de los sujetos colectivos que integran la institución escolar/académica. De este modo, (Sordi \& Ludke, 2009, p. 34) afirman que:

Sendo la evaluación institucional participativa un ejercicio de relectura de la realiadad escolar a partir de sus actores locales, apoyado en distintas eviddencias, pareciéndonos que la parada obligatoria y sistemática inherente al proceso de evaluación posibilita que estos actores vuelvan a se reconocer como colecticvo y como colectivo se interroguen acerca del proyecto que pretenden construir y como colectivo desafien y se amaparen para el trabajo arduo que los espiera, se de facto se ponieren a servicio del aprendizaje de los niños.

Delante del tamiz de la EI,su papel atravesa la afirmación de que al se tener el diagnóstico de los problemas de la institución, los sujetos colectivos (gestores) deben actuar en función del programa de desarrolllo institucional, bien como del proyecto político pedagógico y aún en razón de la adquisión del conocimiento de los estudiantes.

\section{La Evaluación en Amplia Escala: ¿Calidad de la Educación;}

Al profundizar en esta otra especificidad de evaluar el aprendizaje de los estudiantes, tenemos como instrumentos el Examen Brasil, el examen ncional de la enseñaza media, el examen nacional de enseñaza superior y el programa internacional del sistema de evaluación, resultados que corresponden al correlato de la educación como la de mejor calidad, sendo aquella que queda adentro de la media. Así, se destaca el índice del desarrollo de la educación básica (IDEB).

Las evaluaciones en amplia escala son ejecutadas en Brasil a través del Sistema de Evaluación de la Educación Básica (SAEB), lo cual es compuesto por la Evaluación Nacional de la Educación Básica (ANEB) y por la Evaluación Nacional del Rendimiento Escolar (ANRESC); el Examen Nacional de Certificación de Competencias de Jóvenes y Adultos (ENCCEJA); la Prueba Brasil; el Examen Nacional de la Enseñaza Media (ENEM); y la Pruebita Brasil. Estas evaluaciones son instrumentos que visan ejercitar políticas educacionales que atravesan la calidad de la educación. Pues los resultados permiten que los agentes de la educación puedan tomar deciosiones para intervenir en la mejoría de los procesos educativos, infraestructuras y formaciones continuada para se alcanzar la calidad educacional, tenendo en vista que:

Mismo que se considere la realización de evaluación externa sobre los resultados por las escuelas un importante indicador para que los gestores de los sistemas de enseñaza puedan corregir problema y reorientar decisiones y recorridos institucionales, las metodologías que descosideran la diversidad cultural que penetran las redes de escuelas no captan la efectiva dinámica de las unidades escolares, con base sólamente en los resultados finales obtidos por ensayos padronizados (Martins, 2001, p.34).

De esta hecha, resaltamos que corresponde a los gestores tomaren decisiones que son provocadas por medio de la evaluación en amplia escala. La cual senãla los problemas y dificultades que la institución escolar afronta, para allá del rendimiento escolar de los estudiantes.

Desde 1988 que pasa a existir de evaluación en amplia escala en la Educación Básica, a través del Sistema Nacional de Evaluacion de la Enseñaza Publica (SAEP), pues los organismos internacionales tienem interés en la calificación de la mano de obra, por eso que de algún modo hay intervención, pues tiene cierta: "paternidad de los agentes internos sobre este sistema de evaluación" (Bonamino, 2002, p.94). De esta manera, tan sólamente, en 1990, descentraliza la evaluación en amplia escala 
para estados y municipios y, en 1992, el Instituto Nacional de Investigaciones y Estudios Educacionales Anísio Teixeira (INEP) asume la evaluación y, en 1993, hay el $2^{\circ}$ ciclo de evaluación en amplia escala. Así, fue convocada la participación de la gestión escolar, curículo y docencia de Universidades para analizar el sistema de evaluación, buscando así legitimidad académica y reconocimiento social (Bonamino, 2002, p.100). Delante de la necesidad de legitimidad y reconocimiento social, la ALE provoca el financiamiento del Banco Mundial, eso en 1995, lo que implicó cambios en la forma de la administración de la evalución, pasando los estados y municipios a seren aplicados sólamente de la evaluación en amplia escala, dando énfasis a los estudiantes del 4 y $8^{\circ}$ años de la Enseñaza Fundamental y de $3^{\circ}$ año de la Enseñaza Media.

Con la promulgación de la LDB 9.394/96, en su artículo 87, es aboradada la necesidad de una evaluación nacional a partir de una evaluación nacional, he aquí el enunciado:

Art. 87. Es instituido la Década de la Educación, a empezarse un año a partir de la publicación de esta Ley. [...] § $3^{\circ}$. Cada Municipio y, supletivamente, el Estado y la Unión, deberá: [...] IV - integrar todos los establecimientos de Enseñaza Fundamental de su territorio al sistema nacional de evaluación del rendimiento escolar (BRASIL, 1996).

De este modo, la evaluación en amplia escala debe ser un instrumento sistematizado en régimen de colaboración por el ente federado para que abrace la realiadad educacional como un todo, sobretodo, en relación a el aprendizaje de las áreas de portugués y matemática.

En la década de 1990, la evaluación en amplia escala fue cada vez más se sistematizando. De tal manera que, en 2001, es contemplada en el Plano Nacional de Educación con la seguiente finalidad:

De esta manera, podrarse consolidar un sistema de evaluación indispensable para averiguar la eficacia de las políticas públicas en materia de educación. La adoptación de ambos los sistemas [de información y de evaluación] exigir la formación de recursos humanos calificados y la informatización de los servicios, incialmente de las secretarías, pero con el objetivo de integrarlas en red con sus escuelas y con el MEC (BRASIL, 2010, subrayos del autores).

Es importante decir que la evaluación en amplia escala tiene como finalidad averiguar la eficácia de las políticas públicas educacionales, pues delante de los resultados obtidos se encuentra la matriz del problema que implica la falta de calidad de la educación como la necesidad de recursos humanos calificados. Así sendo, está puesto en el documento que,

38. Consolidar y perfeccionar el Sistema Nacional de Evaluación de la Educación Básica - SAEB - y censo escolar.[...] 39. Establecer, en Estados, en cinco años, con la colaboración técnica y financiera de la Unión, un programa de evaluación de desempeño que logre, por lo menos, todas las escuelas de más de 50 alumnos de la ensenãza fundamental y media. [...] 40. Establecer, en los Municipios, en cinco años, programas de acompañamiento y evaluación de los establecimientos de educación infantil. 41. Definir padrones mínimos de calidad del aprendizaje en la Educación Básica en un Conferimiento Nacional de Educación, que englobe la comunidad educacional (BrasiL, 2001).

Las metas son establelecidas de acuerdo con las acciones que deben ser realizadas en favor del perfeccionamiento del sistema nacinal de evaluación de la educación básica, lo que se hace necesaria la dimensión financiera, técnica y programas de evaluación de desempeño que confirme con los resultados que son analizados y diagnosticados como puerta de acceso para cambios estructurales en el sistema de enseñaza.

De este modo, comprendemos que la evaluación en amplia escala es un instrumento potencial que atravesa acciones de planificación para el complejo educacional, la cual objetiva la calidad de la educación a través de las atitudes que son desarrolladas tras el diagnóstico realizado de la situación del contexto escolar. 
Por lo tanto, la evaluación en amplia escala, tal como está puesto por Lima (2006), tiene atribuido un pretenso grado de autonomía a las escuelas, principalmente lo que se refiere a la gestión escolar y a la calidad de la enseñaza, representada por el control de los resultados obtidos por las evaluaciones en amplia escala, regidas por el principio de la eficacia y eficiencia, lo que acarreta en la lejanidad entre el sujeto y el objeto de la evaluación. Reproducindo en el espacio escolar lo que Perrenoud (1999) llama de "Hierarquías de Excelencia", hieraquías estas que regulan y controlan tanto las acciones de quién enseña cuanto de quién aprende. Comprendemos, así que no es tan sólamente un instrumento de control, pero que favorece para un panorama del contexto escolar, tornase un factor importante para el desarrollo educacional a respeto de la gestión escolar y la gestión educacional.

\section{Consideraciones}

Como fue posible identificar al abordarmos la perspectiva triádica de la evaluación, en que concierne a el aprendizaje, institucional y de amplia escala. En el conjunto de las cosas dichas y escritas sobre lo que está puesto, señalamos que la evaluación es un instrumento de tomada de decisión por medio de la planificación sistemática tanto a respeto de la gestión escolar (procesos pedagógicos) cuanto de la gestión educacional (administrativa, financiera y políticas educacionales), o sea, la evaluación es fundamental para que los gestores en el contexto escolar puedan tomar conocimiento del todo de una institución, sea ella escuela o universidad.

De este modo, en los haces de relaciones que son/están enlazados en la evaluación del aprendizaje, sabemos que esta ejerce una función triádica, sendo ella: diagnóstica, sumativa y formativa, se sobresalindo en este enlance la evaluación diagnóstica que se ocupa de la apropriación del nivel de aprendizaje de los estudiantes y la formativa que viabiliza un procedimiento continuo de adquisición y sistematización del conocimiento por medio de un monitoramiento flexible y democrático, con uso de recursos evaluativos diversos, sendo, por lo tanto, una evaluación democrática. Por tanto, comprendemos que dentro de la especificidad evaluativa en el área del aprendizaje la mejor manera de monitorizar es con el diagnóstico y con el proceso continuo de sistematización, por medio de observaciones, participación, trabajos, informes, portfolios, registros diversos que al seren analizados promuevan la ascensión del aprendizaje cúmulativamente y no de forma estanque o paradigmática.

$\mathrm{Al}$ inclinarmos acerca de la evaluación institucional, señalamos que es un instrumento de la gestión educacional que viabiliza la construcción de políticas educacionales, o sea, es una evaluación ampliada del contexto escolar/académico, promoviendo así, el conocimiento de la realidad total de la institución. De tal manera que viabiliza diagnosticar y tomar decisiones que promuevan cambios en la institución, en la infraestructura, en el currículo, en los procesos de enseñaza aprendizaje, así como refleja sobre la necesidad de la formación continuada de los profesores. Considérase que es un instrumento esencialmente democrático por requerir la participación de todos, indicando los elementos estructurales que deben ser reorientados, por eso la importancia de la comisión propria de la evaluación que se ocupe en hacer una autoevaluación institucional, analizar las informaciones colectadas y formar estrategias que son concernientes para los cambios necesarios en el contexto escolar.

La evaluación en amplia escala es un instrumento que viene de fuera de la escuela, o sea, son las secretarías educacionales y el Ministerio de la Educación que se ocupan en evaluar el aprendizaje de los estudiantes, primando por los saberes en las áreas de matemática y portugués, pues son los conocimientos elementares que promuevan la calificación de la mano de obra barata, tenendo en vista que los Organismos Internacionales están por detrás de la evaluación en amplia escala, como el Banco Mundial. Otro elemento importante es que el resultado de la evaluación que corresponde al índice de desarrollo de la educación básica que está mezclada con la calidad de la educación. Dicho de outra manera, la calidad de la educación corresponde la media que la escuela saca en las pruebas, como el Examen Brasil, Evaluación Nacional de la Alfabetización y 
Examen Nacional de la Enseñaza Media. Con el resultado de estas evaluaciones, los gestores tienen los indicadores que atravesan las necesidades de la escuela/institución.

Ya que hacemos inferencia de que las formas de evaluación corresponden a los procesos de la gestión escolar y de la gestión democrática. Instrumento esencial que va allá del control de manera burocrática, pues favorece una tomada de decisión y un plano de acción que pueda promover los cambios necesarios en la institución escolar. De todo modo, la evaluación del aprendizaje, de la institución y en amplia escala objetivan no sólamente el aprendizaje de los estudiantes en el contexto escolar, pero hace con que haya un conocimiento pedagógico, administrativo y financiero correspondente a la planificación de formulación de políticas educacionales.

De esta manera, las formas de evaluar se amplian en el contexto escolar mirando la mejoría y la calidad de la educación. Porque tratar de la evaluación es perspectivar procesos democráticos que deben atravesar la gestión eucacional, tenendo en vista que las competencias y habilidades inherentes al contexto escolar deben ser adquiridas, sin embargo, se hace igualmente necesario que el equipo pedagógico esté subvencionado con formación continuada, con infraestructura adecuada, con recursos materiales disponibles para que la calidad de la educación sea de naturaleza calitativa y no sólamente cantitativa, tenendo en vista que el índice del desarrolllo de la educación básica que mide la calidad de la educación es por media alcanzada de las escuelas, aunque, ni siempre la cantidad de los números en media correspondan la calidad de la educación en el contexto educacional.

Destacamos, por tanto, que la evaluación del aprendizaje, institucional y en amplia escala cumplen con el papel de se obtener un diagnóstico panorámico de la educación, lo que que refleja en la tomada de decisión a través de planificación direccionada para intervenir propósitadamente en las acciones necesarias de mejoría en el contexto escolar/institucional.

Destacamos que con base en lo comentado aquí, no hemos agotado el análisis, pues es necesario profundizar en los aspectos epistémicos de las políticas educativas que la evaluación a gran escala puede favorecer en el ámbito educativo. Para que podamos enfocarnos en el Índice de Desenvolvimento da Educação Básica (IDEB), el financiamiento educativo y la formación docente para trabajar mejor con el sistema de evaluación nacional.

\section{Referências}

Balzan, N. C. \& Dias Sobrinho, J. (1995). Evaluación Institucional: teoría y experiências. Ed. Cortez.

Bonamino, A. (2002). Tiempos de evaluación educacional. Ed. Quartet.

Brasil. (1996). Decreto de Ley no . 9.394, de 20 de diciembre de 1996. Establece las diretrices y bases de l educación nacional. Diario Oficial [de la] República Federativa de Brasil, Brasília, DF, 23 dic.

Carvalho, A. O. P., \& Ribeiro, A. P. de M. (2020). The policy of evaluation of teaching performance in the brazilian context: a case study of the evaluation of teacher performance in the county of Horizonte/CE. Research, Society and Development, 9(4), e155943024. https://doi.org/10.33448/rsd-v9i4.3024

Mika, N. M. (2008). La evaluación institucional como instrumento de mejoría del proceso enseñaza-aprendizaje. Cuaderno Pedagógico. SEED/PDE/Universidade Estadual de Ponta Grossa - UEPG. http://www.diaadiaeducacao.pr.gov.br/portals/pde/arquivos/35-2.pdf .

Carminatti, S. S. H. \& Borges, M. K. (2012). Perspectivas de la evaluación del aprendizaje en la contemporaneidad. Estudios en la Evaluación Educacional. San Pablo. 23(52), 160-78. http://www.fcc.org.br/pesquisa/publicacoes/eae/arquivos/1734/1734.pdf.

Décia, A. C. M. \& Argollo, R. S. N. (2010). Autoevaluación institucional de la UFBA en la era Sinaes: la experiencia revisitada en la Escuela de Administración. Estudios en la Evaluación Educacional. 21(46), 387- 404.

Fernandes, M. E. A. (2001) Progestión: ¿como desarrollar la evaluación institucional en la escuela ¿ Módulo IX, Brasília: CONSED.

Ferreira, N. S. C. (2003) Repensando y resignificando la gestión democrática de la educación en la "cultura globalizada". In: Revista Educación \& Sociedad. Campinas, 25(89), 1227-1249.

Foulcault, M. A Arqueologia do Saber. 7. ed. Rio de Janeiro: Forense Universitária, 2008[1969]. Tradução de: Luiz Felipe Baeta Neves.

Freitas, L. C. et al. (2009). Evaluación educacional: caminhando por la contramano. Vozes.

Gadotti, M. (2000). Perspectivas actuales de la educación. Ed. Artes Médicas. 
Research, Society and Development, v. 11, n. 1, e40211125171, 2022

(CC BY 4.0) | ISSN 2525-3409 | DOI: http://dx.doi.org/10.33448/rsd-v11i1.25171

Gatti, B. A. (2006). Evaluación Institucional: ¿proceso descriptivo, analítico o reflexivo ¿ Estudios en la Evaluación Educacional, 17(34), 7-14,

Griboski, C. M. (2012). El Enade como inductor de la calidade de la educación superior. Estudios en la Evaluación Educacional, 23(53), 178-195.

Hadji, C. (2001) Avaliação desmistificada. Artmed.

Hoffmann, J. M. L. (1998). Pontos e contrapontos: do pensar ao agir em avaliação. Mediação.

Hoffmann, J. M. L. (2009). Evaluar para promover: las flechas del camino. Mediação.

Libâneo, J. C. (1994). Didáctica.: Cortez.

Lima, L. (2006) Compreender a Escola: Perspectivas de análise organizacional. ASA.

Luckesi, C. C. (2005). Avaliação da aprendizagem escolar: estudos e proposições. (17a ed.), Cortez.

Luckesi, C. C. (2012). Evaluación del aprendizaje escolar: estudios y proposiciones. (22a ed.), Cortez.

Machado, M. A. C. A. (1995) Diagnóstico para superar o tabu da avaliação nas escolas. AMAE Educando, 255.

Martins, A. M. (2001). A descentralização como eixo das reformas do ensino: uma discussão da literatura. Educação \& Sociedade. Revista de Ciência da Educação: CEDES, n. 77, ano XXII, 28-48.

Masetto, M. T. (1990). Evaluación Institucional - definiciones y posicionamientos. Estudios en Evaluación Educacional, 9-12.

Perrenoud, P. (2008) Avaliação - Da excelência à regulação das aprendizagens - entre duas lógicas. Artes Médicas.

Perrenoud, P. (1999). Avaliação: da Excelência à Regulação das Aprendizagens. Artmed Editora.

Ribeiro, E. A. (2012). Convergências e divergências das práticas de autoavaliação institucional em IES pública e privada na percepção da comunidade acadêmica. Estudos em Avaliação Educacional, 23(52), 168-189.

Saul, A. M. (1990). Evaluación de la Universidad: buscando una alternativa democrática. Estudios en Evaluación Educacional, (1), 17-20.

Sordi, M. R. L. de, \& Ludke, M. (2009). De la Evaluación del Aprendizaje a la Evaluación Institucional: aprendizajes necesarios. Evaluación, 14(2) 313-336, jul.

Souza, Â. R. de, \& Dittrich, D. D. (2012). Evaluación en la escuela, evaluación de la escuela: análisis de una experiencia escolar. Estudios en Evaluación Educacional, 23(52), 26-46 